\title{
Evaluación y manejo del adulto inmunocompetente hospitalizado por neumonía adquirida en la comunidad, en un hospital de baja complejidad, basado en la G uía Clínica Chilena
}

\author{
Luis Manuel Sanhueza $A^{1 a}$, Cristián Vásquez $P^{2}$, \\ Fabiola Sepúlveda $Z^{3}$, Francisca Barahona $C^{4 b}$, \\ Rubén G onzález $\mathrm{C}^{5 \mathrm{c}}$, Fernando Saldías $\mathrm{P}^{6}$. \\ Management of community acquired \\ pneumonia in adults following clinical \\ guidelines at a rural hospital
}

Background: A National Consensus Guideline published in 2005 established the basis for the diagnostic, severity assessment and treatment of community acquired pneumonia (CAP) in the adult population. The compliance with pneumonia clinical guidelines has been associated to a reduction in hospital stay, healthcare-related costs, morbidity and mortality. Aim: To describe the management and outcome of non-severe CAP in hospitalized adult patients treated in a rural hospital, based on the national clinical guidelines. Patients and methods: Ninety six patients aged $74 \pm 13$ years ( 50 males) hospitalized with non-severe pneumonia (group 3) at a community-based primary care center between January 1, 2006, and March 31, 2007, were evaluated. Results: Eighty percent of patients had concomitant diseases such as hypertension in $49 \%$, diabetes in $23 \%$ and chronic obstructive pulmonary disease in $18 \%$. All were treated with a third generation cephalosporin (ceftriaxone 1-2 g/day IV) as empirical therapy. Only 9\% of patients also received a macrolide. Early switch to oral antimicrobial therapy was successful in two third of cases. Mean hospital length of stay was $5.0 \pm 2.5$ days, and 30-day mortality was $6.3 \%$. Conclusions: Following the recommendations of the national clinical guidelines, most of these patients had a favorable response to monotherapy with a ß-lactam antimicrobial. Early switch therapy to oral antibiotic was effective and safe, reducing significantly hospital length of stay as compared to previous national clinical studies (Rev Méd Chile 2009; 137: 1283-90).

(Key words: Anti-bacterial agents; Pneumonia, bacterial; Standards)

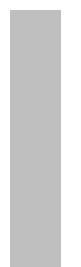

\footnotetext{
Recibido el 4 de marzo, 2009. Aceptado el 10 de septiembre, 2009.

Facultad de Medicina, Pontificia Universidad Católica de Chile ${ }^{1}$, Hospital de Puerto Aysén, Servicio de Salud de Aysén ${ }^{2}$, Hospital de Licantén, Servicio de Salud del Maule ${ }^{3}$. Facultad de Medicina, Universidad de Chile ${ }^{4}$, Universidad de La Frontera, Temuco ${ }^{5}$, Departamento de Enfermedades Respiratorias, Facultad de Medicina, Pontificia Universidad Católica de Chile, Santiago de Chile ${ }^{6}$

${ }^{a}$ Becado de Medicina Interna

${ }^{b}$ Becada de Medicina General Familiar

'Interno de Medicina
}

Correspondencia a: Dr. Fernando Saldías Peñafiel. Departamento de Enfermedades Respiratorias, Pontificia Universidad Católica de Chile. Marcoleta $352-1^{\text {er }}$ piso, Santiago, Chile. Teléfonos: 6331541 - 3543242. Fax: 6335255. E mail: fsaldias@med.puc.cl 
$\mathrm{E}^{n}$ n Chile, la neumonía es la principal causa de complicaciones y muerte por enfermedades infecciosas y respiratorias en el adulto ${ }^{1}$. La tasa de mortalidad por neumonía en adultos entre 20 y 64 años es 14,8 por 100.000 habitantes, pero se eleva dramáticamente en los adultos mayores de 65 años, con una letalidad de 6,6 individuos por cada 1.000 habitantes $^{2}$.

En estudios poblacionales, se ha estimado que alrededor de $70 \%$ a $80 \%$ de los episodios de neumonía adquirida en la comunidad (NAC) son tratados en el ámbito ambulatorio, dado el bajo riesgo de complicaciones y muerte (letalidad inferior a 1\%$2 \%$ ) y sólo $20 \%$ a $30 \%$ requiere manejo hospitalario por la gravedad del cuadro, concentrándose en esta población el mayor riesgo de complicaciones, muerte y demanda de recursos de salud ${ }^{3-5}$. Este último grupo corresponde principalmente, a adultos mayores de 65 años o portadores de enfermedades crónicas.

No obstante lo anterior, se ha observado una gran variabilidad en la tasa de hospitalizaciones en diferentes áreas geográficas, posiblemente atribuibles al empleo de diferentes criterios clínicos para categorizar la gravedad de los enfermos y decidir el lugar de manejo (ambulatorio u hospitalario) según las características de los sistemas de salud ${ }^{6-11}$. En atención a esto, a nivel internacional se han generado distintas iniciativas dirigidas a la elaboración de recomendaciones y guías clínicas para sistematizar la evaluación y manejo de los pacientes con neumonía comunitaria, tales como las guías clínicas de la Sociedad Americana de Tórax y Sociedad Americana de Enfermedades Infecciosas (Guía ATS/IDSA 2001/03/07) o la Sociedad Británica de Tórax (Guía BTS 2001/04/09) ${ }^{12-18}$. En 1999 se confeccionó la primera guía para manejo de la NAC en Chile, elaborada por un grupo de expertos de la Sociedad Chilena de Enfermedades Respiratorias, quienes adaptaron la experiencia extranjera a nuestro sistema de salud ${ }^{19}$.

Debido a cambios epidemiológicos y tecnológicos acontecidos en los últimos años y a la aparición de nuevas publicaciones sobre el tema en el medio nacional, un comité de expertos de las Sociedades Chilenas de Enfermedades Respiratorias y Enfermedades Infecciosas examinó la evidencia disponible y elaboró un documento de consenso el año 2005 para la evaluación y manejo de la neumonía comunitaria del adulto ${ }^{20}$. El Consenso Chileno de Neumonía propone criterios clínicos y radiográficos para evaluar el riesgo de complicaciones y muerte de los enfermos en la evaluación inicial, distingue cuatro categorías o grupos de riesgo, dos grupos de manejo ambulatorio (grupos 1 y 2) y dos de manejo hospitalario (grupos 3 y 4). En los pacientes del grupo 3, de gravedad moderada, se recomienda su manejo inicial en sala de cuidados generales, tratamiento antibiótico con agentes ß-lactámicos vía intravenosa y cambio precoz a la vía oral al alcanzar los criterios de estabilidad clínica ${ }^{20}$.

En nuestro país disponemos de escasa información acerca de la implementación de las guías clínicas de neumonía y sus resultados en términos de evolución y pronóstico ${ }^{21,22}$. Así, el principal objetivo de nuestro estudio fue describir el manejo de pacientes adultos inmunocompetentes hospitalizados por neumonía comunitaria de gravedad moderada (grupo 3) basados en la guía clínica chilena en un hospital de baja complejidad.

\section{PACIENTES Y MÉTODOS}

Se evaluaron 96 pacientes adultos inmunocompetentes hospitalizados por un episodio de neumonía comunitaria en el Servicio de Medicina del Hospital de Yumbel, región del Bío-Bío, entre enero de 2006 y marzo de 2007. Los pacientes cumplieron los criterios diagnósticos de neumonía descritos por Fang y $\mathrm{cols}^{23}$, y los criterios de hospitalización en sala de cuidados generales (grupo 3) descritos en la Guía Clínica Chilena de Neumonía ${ }^{20}$. Se excluyeron del estudio los pacientes portadores del virus de inmunodeficiencia humana, tumores sólidos o hematológicos en quimioterapia, neutropenia (leucocitos $<1.000 / \mathrm{mm}^{3}$ ), tratamiento quimioterápico o inmunosupresor, o tratamiento con dosis $\geq 20 \mathrm{mg} /$ día de prednisona o su equivalente durante por lo menos un mes dentro de los seis meses anteriores a la consulta; y los pacientes con criterios de neumonía comunitaria grave ${ }^{13,20}$, quienes fueron derivados al Hospital Base de Los Ángeles. Además, se excluyeron del estudio los pacientes hospitalizados en el servicio de medicina por neumonía comunitaria ( $\mathrm{n}=41$ ) que no fueron manejados siguiendo las recomendaciones de la Guía Clínica Chilena de Neumonía ${ }^{20}$.

El Hospital de Yumbel es un centro asistencial de baja complejidad (tipo 4) localizado en la región del Bío-Bío, pertenece al Servicio de Salud Bío-Bío, atiende a una población asignada de 48.940 habitan- 
tes provenientes de las comunas de Yumbel y Cabrero, el Servicio de Medicina está dotado de 32 camas, dispone de red de oxígeno y laboratorio básico, y el equipo de salud encargado de la atención cerrada está compuesto por cinco médicos generales, dos enfermeras, un kinesiólogo y una nutricionista.

Los pacientes fueron manejados siguiendo las recomendaciones de la Guía Clínica Chilena de Neumonía (2005), en relación a: a) Evaluación de la gravedad y criterios de hospitalización, b) Tratamiento antibiótico empírico, c) Evaluación y seguimiento en el hospital, d) Criterios de fracaso de tratamiento, e) Cambio precoz a antibioterapia oral y alta precoz ${ }^{20}$.

En los pacientes hospitalizados por neumonía comunitaria se consignaron los siguientes antecedentes clínico-demográficos: edad, género, enfermedades preexistentes ${ }^{24}$ (se examinaron las comorbilidades descritas en las guías clínicas de la ATS y BTS ${ }^{15,16}$ ), cuadro clínico y radiográfico, signos vitales y estado de conciencia en la admisión al hospital, comorbilidades descompensadas y motivo de hospitalización. El compromiso cualitativo de conciencia o confusión mental fue definido por la presencia de desorientación en tiempo, espacio o en relación a personas que no correspondía a una condición basal conocida o coma. La alteración de los signos vitales en la admisión al hospital fue definida por la presencia de a) frecuencia cardiaca mayor de 120 lat $/ \mathrm{min}, \mathrm{b})$ presión arterial menor de $90 / 60 \mathrm{mmHg}$, c) frecuencia respiratoria mayor de 20 $\mathrm{resp} / \mathrm{min}$ y d) temperatura axilar mayor de $40^{\circ} \mathrm{C}$.

En todos los pacientes se realizó una radiografía de tórax en dos proyecciones (PA y lateral) al ingreso al hospital. La radiografía fue informada e interpretada por el médico tratante y uno de los investigadores del estudio (LMS). El patrón radiográfico fue clasificado como imagen de relleno alveolar, patrón intersticial o patrón mixto. Además, se consignó el grado de extensión del compromiso radiográfico (unilobar, multilobar, bilateral) y se registró la presencia de derrame pleural, absceso o cavitación.

Finalmente, se consignó la terapia antibiótica inicial, evolución clínica, complicaciones intrahospitalarias, modificaciones del tratamiento, posibilidades de cambio precoz a antibioterapia oral, duración de la estadía hospitalaria y letalidad en el seguimiento a 30 días. El criterio de curación en el seguimiento a 30 días fue definido en términos clínicos: mejoría significativa o desaparición de los síntomas respiratorios o fiebre, sin necesidad de modificar el tratamiento antimicrobiano empírico inicial o ser readmitido en el hospital. Se consideró fracaso en el cambio precoz a la terapia antibiótica oral cuando los pacientes no alcanzaron los criterios de estabilidad clínica descritos en el Consenso Chileno de Neumonía ${ }^{20}$ después del tercer día de hospitalización.

Los resultados se expresan como valores promedio \pm desviación estándar para las variables medidas en escala numérica y en porcentaje para las medidas en escala nominal. Las variables cualitativas (antecedentes clínico-radiográficos, evolución clínica y letalidad) fueron comparadas mediante la prueba de Chi cuadrado y las variables continuas con la prueba t de Student. Las diferencias entre las variables fueron consideradas significativas con un valor de $\mathrm{p}<0,05$.

\section{Resultados}

Características clínicasy demográficas en la admisión al hospital. En el periodo del estudio se evaluaron 96 pacientes, edad promedio de 74,4 $\pm 13,1$ años (rango: 16-95), la mayoría eran adultos mayores de 65 años (82\%) y de procedencia rural (Tabla 1). El 80,2\% de los pacientes tenía enfermedades preexistentes, especialmente hipertensión arterial (49\%), diabetes mellitus (22,9\%), enfermedad pulmonar obstructiva crónica $(17,7 \%)$ e insuficiencia cardiaca $(12,5 \%)$. Las comorbilidades fueron más frecuentes en la población senescente ( $85,1 \%$ vs $63,6 \%$; p < <, 05) y sólo $20 \%$ de los pacientes hospitalizados por neumonía comunitaria no tenía comorbilidades.

Los principales criterios de hospitalización en esta cohorte de pacientes con neumonía comunitaria fueron la edad avanzada (82\%), presencia de comorbilidades (80\%), anomalías de los signos vitales (45\%), compromiso radiográfico multilobar o bilateral (40\%), falla respiratoria hipoxémica (20\%) y compromiso de conciencia $(5,2 \%)$. No hubo hospitalizaciones debido a problemas psicosociales, trastornos psiquiátricos o falta de red de apoyo social.

Manejo de la neumonía comunitaria en el hospital. Siguiendo las recomendaciones de la guía clínica nacional de neumoní $a^{20}$, todos los pacientes fueron manejados con hidratación parenteral en las primeras $24 \mathrm{~h}$, corrección de la hipoxemia y tratamiento antimicrobiano vía intravenosa (ceftriaxona 1-2 g/día), en nueve pacientes se adicionó un macrólido (claritromicina $500 \mathrm{mg}$ cada $12 \mathrm{~h}$ ) a las $72 \mathrm{~h}$ debido a 


\section{Tabla 1. Características clínicas y demográficas de 96 pacientes hospitalizados por neumonía adquirida en la comunidad de gravedad moderada (G rupo 3 ) en el H ospital de Yumbel}

\begin{tabular}{|lll|}
\hline Edad (años) $(\mathrm{X} \pm \mathrm{DE})$ & $74,4 \pm 13,1$ & (rango: 16-95) \\
Sexo masculino (\%) & $50 / 96$ & $(52,1 \%)$ \\
Comorbilidad (\%) & $77 / 96$ & $(80,2 \%)$ \\
Hipertensión arterial & $47 / 96$ & $(49 \%)$ \\
Diabetes mellitus & $22 / 96$ & $(22,9 \%)$ \\
EPOC & $17 / 96$ & $(17,7 \%)$ \\
Cardiopatía-ICC & $12 / 96$ & $(12,5 \%)$ \\
Asma bronquial & $5 / 96$ & $(5,2 \%)$ \\
Secuela de AVC & $5 / 96$ & $(5,2 \%)$ \\
Neoplasia & $4 / 96$ & $(4,2 \%)$ \\
Daño hepático crónico & $3 / 96$ & $(3,1 \%)$ \\
Insuficiencia renal crónica & $2 / 96$ & $(2,1 \%)$ \\
Criterios de gravedad al ingreso & & \\
Edad $>65$ años & $79 / 96$ & $(82,3 \%)$ \\
Anomalía signos vitales & $40 / 88$ & $(45,4 \%)$ \\
Confusión mental & $5 / 96$ & $(5,2 \%)$ \\
NAC multilobar-bilateral & $39 / 96$ & $(40,6 \%)$ \\
Derrame pleural & $3 / 92$ & $(3,3 \%)$ \\
Hipoxemia $\left(\mathrm{SaO}{ }_{2}<90 \%\right)$ & $17 / 84$ & $(20,2 \%)$ \\
BUN $>20$ mg/dL & $8 / 17$ & $(47,1 \%)$ \\
Leucopenia $<4.000 / \mathrm{mm}^{3}$ & $1 / 32$ & $(3,1 \%)$ \\
\hline
\end{tabular}

Nota: El número del denominador corresponde a los pacientes con la variable registrada.

EPOC: enfermedad pulmonar obstructiva crónica; ICC: insuficiencia cardiaca congestiva; AVC: accidente vascular cerebral; NAC: neumonía adquirida en la comunidad; BUN: nitrógeno ureico sérico. Anomalía de signos vitales: FC $>120 \mathrm{lat} / \mathrm{min}, \mathrm{PA}<90 / 60 \mathrm{mmHg}$, FR $>20 \mathrm{resp} / \mathrm{min}$ o $\mathrm{T}>40^{\circ} \mathrm{C}$.

evolución clínica insatisfactoria (mejoría clínica lenta o deterioro clínico progresivo). La estadía en el hospital fue más prolongada en los pacientes que se agregó un macrólido al tratamiento inicial con ceftriaxona $(8,0 \pm$ 3,6 vs $4,6 \pm 2,1$ días; $\mathrm{p}<0,001$ ). El cambio a terapia antibiótica oral ocurrió en promedio a los 3,0 \pm 1,8 días (rango: 1-10 días) y en dos tercios de los pacientes
(61/90) aconteció dentro de los primeros tres días (Tabla 2). En el cambio a antibioterapia oral, se prescribió amoxicilina con ácido clavulánico (875/125 $\mathrm{mg} \mathrm{c} / 12 \mathrm{~h}$ ó 500/125 mg c/8 h) en todos los pacientes. Sólo un paciente fue dado de alta sin cambio a antibiótico oral, completando 7 días de tratamiento con ceftriaxona intravenosa dentro del hospital.

\section{Tabla 2. Tratamiento antimicrobiano prescrito a 96 pacientes hospitalizados por neumonía adquirida} en la comunidad de gravedad moderada en el H ospital de Yumbel
Tratamiento antimicrobiano
Ceftriaxona $1-2 \mathrm{~g} /$ día iv
96/96
$(100 \%)$
Claritromicina $500 \mathrm{mg}$ cada $12 \mathrm{~h}$
$9 / 96$
$(9,3 \%)$
Tratamiento antibiótico iv $(\mathrm{X} \pm \mathrm{DE})$
$3,0 \pm 1,8$ días
(rango: 1-10 días)
Cambio a antibioterapia oral $\leq 3$ días
$61 / 90$
$(67,8 \%)$
Fracaso cambio precoz a antibiótico oral
$29 / 90$
$(32,2 \%)$ 
Los pacientes que sobrevivieron al episodio de neumonía alcanzaron los criterios de estabilidad clínica descritos en la guía clínica chilena y fueron dados de alta dentro de las siguientes $24 \mathrm{~h}$, lo cual ocurrió en promedio a los 5,0 $\pm 2,5$ días (rango: 1-14 d). Cuarenta y dos pacientes (47\%) fueron dados de alta dentro de los primeros cuatro días de hospitalización, una vez alcanzados los criterios de estabilidad clínica sugeridos en el consenso chileno (Tabla 3). Seis pacientes fallecieron en el hospital debido a evolución clínica desfavorable después del primer día de hospitalización (letalidad: 6,25\%), de los cuales, tres fueron derivados con criterios de neumonía comunitaria grave (grupo 4) para manejo en la Unidad de Cuidados Intensivos del Hospital de Los Ángeles y tres pacientes fallecieron dentro del primer día en el Hospital de Yumbel, asociado a deterioro clínico rápidamente progresivo, y no fueron trasladados por presentar comorbilidades avanzadas (neoplasia diseminada o daño hepático crónico severo). No hubo diferencias significativas en la edad $(80,7 \pm 14,9$ vs $74,0 \pm 12,9$ años; p: 0,22 ), género ( $50 \%$ vs $52 \%$ varones; p: 0,91$)$ y presencia de comorbilidad $(83,3 \%$ vs 78,9\%; p: 0,79$)$ entre los pacientes fallecidos y sobrevivientes en el seguimiento a 30 días. En cambio, la anomalía de los signos vitales ( $83,3 \%$ vs $46,6 \% ; \mathrm{p}<0,05)$ y falla respiratoria hipoxémica $(50 \%$ vs $14 \% ; \mathrm{p}<0,05)$ en la admisión al hospital fueron más frecuentes en los pacientes fallecidos.

\section{DisCUSIÓN}

La neumonía adquirida en la comunidad (NAC) es una de las afecciones respiratorias más frecuentes que afectan a la población infantil y adulta, su incidencia anual oscila entre 5 y 13 casos por 1.000 habitantes, es la séptima causa de muerte en nuestro país, especialmente en adultos mayores de 65 años con enfermedades preexistentes, constituyendo un problema relevante de salud pública ${ }^{1-3}$.

Una vez establecido el diagnóstico, una de las decisiones más importantes que debe tomar el médico es el lugar de tratamiento (ambulatorio o en el hospital), la extensión del estudio microbiológico y el tratamiento antimicrobiano basado en criterios de gravedad definidos ${ }^{4}$. En los estudios clínicos se ha demostrado que existe importante variabilidad en la evaluación de la gravedad de los pacientes con neumonía, decisión del lugar de manejo $^{6-8}$, duración de la hospitalización ${ }^{5-8}$ y elección del tratamiento antibiótico ${ }^{9-11}$. Las variaciones observadas en el tratamiento de los pacientes ingresados con neumonía pueden atribuirse a factores relacionados con el propio paciente, siendo la gravedad del caso y la presencia de comorbilidades las variables que tienen mayor impacto en la mortalidad ${ }^{4}$, y otros factores relacionados con la estructura y organización del sistema sanitario, el tipo de hospital (alta o baja complejidad), las características socioculturales de la población atendida y factores relacionados con el personal de salud ${ }^{6-11}$. De hecho, los estudios clínicos han demostrado que los médicos suelen sobreestimar la gravedad de los enfermos con neumonía y tienen dificultades para detectar a los pacientes de riesgo elevado ${ }^{25,26}$. Por ello, a nivel internacional las sociedades científicas han elaborado normativas y guías clínicas para optimizar el manejo de los enfermos, con recomendaciones consensuadas para su diagnóstico, evaluación de la gravedad y tratamiento ${ }^{12-18}$.

\section{Tabla 3. Evolución de 96 pacientes hospitalizados por neumonía adquirida en la comunidad de gravedad moderada en el $\mathrm{H}$ ospital de Yumbel}

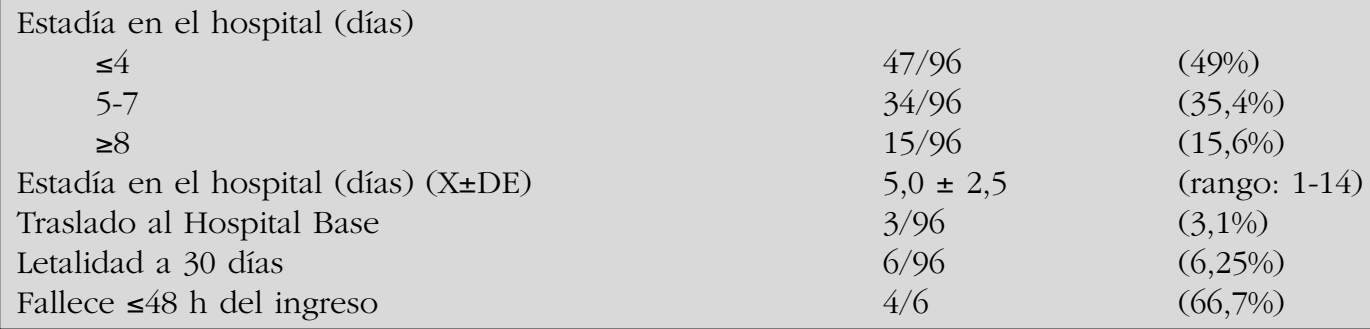


En nuestro país, se han elaborado dos guías clínicas para el manejo de la neumonía comunitaria del adulto inmunocompetente (1999 y 2005), las cuales realizan recomendaciones específicas sobre el diagnóstico, evaluación de la gravedad y tratamiento antibiótico empírico ${ }^{19,20}$. En la Guía Clínica Nacional de Neumonía más reciente, se recomienda evaluar la gravedad de los enfermos, empleando básicamente criterios clínicos y sociodemográficos, para definir el lugar de manejo, la extensión del estudio microbiológico y el tratamiento antibiótico empírico ${ }^{20}$. En los pacientes hospitalizados en sala de cuidados generales se recomienda iniciar tratamiento antibiótico empírico con agentes ß-lactámicos y evaluar la respuesta al tratamiento en forma sistemática basado en elementos clínicos (estado de conciencia, signos vitales, requerimientos de oxígeno, ingesta oral), para decidir el momento de cambio a antibioterapia oral (switch therapy) y considerar las condiciones de egreso hospitalario, lo que permitiría reducir la estadía en el hospital y reducir los costos de la hospitalización comparado con otras series nacionales ${ }^{27-31}$ (Tabla 4). El manejo de los enfermos hospitalizados por neumonía comunitaria basados en las guías clínicas ha demostrado que acorta su estadía en el hospital, disminuye el riesgo de complicaciones y muerte ${ }^{32-36}$.

En nuestro medio, la mayoría de los estudios clínicos publicados corresponden a pacientes con neumonía hospitalizados en centros asistenciales de alta complejidad ${ }^{21,27-31}$ y no existen estudios que hayan evaluado la guía clínica nacional de 2005. En nuestro estudio, evaluamos la evolución clínica de los pacientes adultos con neumonía comunitaria, de gravedad moderada, manejados en la sala de cuidados generales de un hospital de baja complejidad, siguiendo las recomendaciones de la guía clínica chilena de neumonía. Las características clínicas, gravedad y complejidad de los pacientes descritos en este estudio probablemente difieren de aquellos manejados en servicios de alta complejidad de nuestro país ${ }^{21,27-31}$ (Tabla 4). Dado que las características y disponibilidad de recursos varía significativamente entre los distintos servicios de salud, y los pacientes más graves suelen ser manejados en los hospitales de alta complejidad, no es fácil comparar los resultados de los distintos centros asistenciales. Desgraciadamente, en los hospitales rurales de baja complejidad no es factible medir en forma rutinaria los índices de gravedad recomendados en las guías clínicas internacionales ${ }^{12-18}$, el índice de gravedad de Fine o CURB-65, ya que los médicos de atención primaria sólo disponen de laboratorio básico en horario diurno. La evaluación de la gravedad de los pacientes con neumonía comunitaria sugerida en el consenso chileno y el índice CRB-65 permiten estratificar a los pacientes en distintas categorías de riesgo, sin necesidad de exámenes de laboratorio complementarios, lo cual junto con el juicio clínico permitiría decidir el lugar de manejo (ambulatorio, sala de cuidados generales, UCI) y planificar el tratamiento de los enfermos ${ }^{17,18,20}$.

En nuestro estudio, la mayoría de los pacientes manejados según las recomendaciones de la guía clínica chilena (agente ß-lactámico vía EV) evolucionó favorablemente, y en menos de 10\% de los casos fue necesario agregar un macrólido por evolución tórpida

\section{Tabla 4. Estadía en el hospital y letalidad de los pacientes adultos hospitalizados en sala de cuidados generales por neumonía adquirida en la comunidad. Estudios nacionales}

\begin{tabular}{|lccccc|}
\hline Estudios & $\mathbf{1}$ & $\mathbf{2}$ & $\mathbf{3}$ & $\mathbf{4}$ & $\mathbf{5}$ \\
$\mathbf{N}$ & $\mathbf{2 7 1}$ & $\mathbf{1 7 0}$ & $\mathbf{2 0 0}$ & $\mathbf{1 . 0 7 5}$ & $\mathbf{1 6 6}$ \\
\hline Tipo de hospital & Tipo 1 & Tipo 1 & Tipo 1 & Tipo 1 y 2 & Tipo 1 \\
Edad (años) (X \pm DE) & $66 \pm 20$ & 68 & $63 \pm 19$ & $68 \pm 17$ & $66 \pm 18$ \\
Comorbilidad & $67 \%$ & $77,6 \%$ & $74,5 \%$ & $72,4 \%$ & $73 \%$ \\
Estadía en el hospital (X DE) (días) & $7,2 \pm 4,6$ & 9,2 & $12 \pm 9,6$ & $10,9 \pm 9,2$ & $7,4 \pm 5,9$ \\
Letalidad en el hospital & $2,6 \%$ & $11 \%$ & $17,5 \%$ & $14,3 \%$ & $7,2 \%$ \\
\hline
\end{tabular}

Nota: 1. Saldías y cols ${ }^{27} ; 2$. Dintrans y cols ${ }^{28} ; 3$. Riquelme y cols ${ }^{29} ; 4$. Gil y cols ${ }^{30} ; 5$. Díaz y cols ${ }^{31}$. En el estudio de Dintrans y cols 60 pacientes requirieron traslado a una unidad de cuidados especiales durante la evolución en el hospital, y el estudio de Riquelme y cols incluyó 18 pacientes hospitalizados en UCI. 
o falla de tratamiento después del tercer día de admisión. En dos tercios de los pacientes fue factible y seguro cambiar a antibioterapia oral en forma precoz ( $\leq 72 \mathrm{~h})$ y manejar ambulatoriamente después de $24 \mathrm{~h}$ de haber alcanzado los criterios de estabilidad clínica, reduciendo en forma significativa la estadía en el hospital. En el estudio de Ramírez y $\operatorname{cols}^{37}$, dos tercios de los pacientes hospitalizados por neumonía alcanzaron los criterios de estabilidad clínica y fue factible el cambio a la terapia antibiótica oral dentro de los primeros tres días de hospitalización. La estadía media en el hospital de este grupo fue de 3,4 días.

Como ha sido comunicado en la literatura ${ }^{32-36}$, la adherencia a las guías clínicas podría tener un gran impacto en la evolución de los enfermos y en el ámbito económico, al reducir los costos asociados a hospitalizaciones prolongadas y al empleo de esquemas antibióticos de amplio espectro y costo elevado no recomendados en las guías clínicas, a la vez que disminuiría el potencial desarrollo de resistencia bacteriana y efectos adversos asociados a esquemas antimicrobianos de amplio espectro.

\section{REFERENCIAS}

1. Programa de Infecciones Respiratorias Agudas (IRA), Departamento de Estadísticas e Información de Salud, Ministerio de Salud de Chile, 2008. http:// deis.minsal.cl/index.asp.

2. Valdivia G. Epidemiología de la neumonía del adulto adquirida en la comunidad. Rev Chil Infect 2005; 22 (Supl 1): S11-S17.

3. Almirall J, Bolibar I, Vidal J, Sauca G, Coll P, NikLasson B ET AL. Epidemiology of communityacquired pneumonia in adults: a population-based study. Eur Respir J 2000; 15: 757-63.

4. Fine MJ, Smith MA, Carson CA, Mutha SS, Sankey SS, Weissfeld LA, Kapoor WN. Prognosis and outcomes of patients with community-acquired pneumonia. A meta-analysis. JAMA 1996; 275: 134-41.

5. Laurichesse H, Robin F, Gerbaud L, Pochet P, Gourdon F, Beytout J et al. Empirical therapy for nonhospitalized patients with community-acquired pneumonia. Study Group of General Practitioners. Eur Respir J 1998; 11: 73-8.

6. Fine MJ, Singer De, Phelps AL, Hanusa BH, Kapoor WN. Differences in length of hospital stay in patients with community-acquired pneumonia: a prospective four-hospital study. Med Care 1993; 31: 371-80.

7. Feagan BG, Marrie TJ, Lau Cy, Wheeler SL, Wong CJ, VANDERVOORT MK. Treatment and outcomes of community-acquired pneumonia at Canadian hospitals. Can Med Assoc J 2000; 162: 1415-20.
En nuestro medio, son escasos los estudios que examinan el manejo de los pacientes con neumonía comunitaria de manejo ambulatorio ${ }^{38}$ u hospitalizados en centros de baja complejidad. Parece alentador el empleo sistemático y riguroso de las recomendaciones propuestas por el consenso chileno de neumonía, aunque sería deseable establecer estas comparaciones en base a estudios prospectivos, randomizados y controlados, incluyendo nuevas alternativas antibióticas y en una población más grande e idealmente multicéntrica.

\section{Agradecimiento}

Los autores agradecen la valiosa ayuda otorgada por el personal administrativo y paramédico del Hospital de Yumbel en la implementación del estudio. Durante la ejecución del estudio, el Dr. LM Sanhueza se desempeñaba como médico en etapa de destinación y formación en el Hospital de Yumbel; y los Dres. C Vásquez y F Sepúlveda estaban realizando una rotación del internado de medicina de la Universidad de Concepción.

8. Jin Y, Marrie TJ, Carriere KC, Predy G, Houston C, Ness $\mathrm{K}$ ET AL. Variation in management of communityacquired pneumonia requiring admission to Alberta, Canada hospitals. Epidemiol Infect 2003; 130: 41-51.

9. OrtQvist A. Antibiotic treatment of community-acquired pneumonia in clinical practice: a European perspective. J Antimicrob Chemother 1995; 35: 205-12.

10. Huchon GJ, Gialdroni-Grassi G, Léophonte P, Manresa F, SChaberg T, Woodhead M. Initial antibiotic therapy for lower respiratory tract infection in the community: a European survey. Eur Respir J 1996; 9: 1590-5.

11. Gilbert K, Gleason PP, Singer DE, Marrie TJ, Coley CM, OвRоSкy DS et al. Variations in antimicrobial use and cost in more than 2,000 patients with community-acquired pneumonia. Am J Med 1998; 104: 17-27.

12. Bartlett JG, Dowell SF, Mandell LA, File JR TM, Musher DM, FIne MJ. Practice guidelines for the management of community-acquired pneumonia in adults. Infectious Diseases Society of America. Clin Infect Dis 2000; 31: 347-82.

13. Niederman MS, Mandell LA, Anzueto A, Bass JB, Broughton WA, Campbell GD et al. Guidelines for the management of adults with community-acquired pneumonia. Diagnosis, assessment of severity, antimicrobial therapy, and prevention. Am J Respir Crit Care Med 2001; 163: 1730-54.

14. Mandell LA, Bartlett JG, Dowell SF, File TM Jr, Musher DM, Whitney C; Infectious Diseases Society of AMERICA. Update of practice guidelines for the management of community-acquired pneumonia in im- 
munocompetent adults. Clin Infect Dis 2003; 37 : 1405-33.

15. Mandell la, Wunderink RG, Anzueto A, Bartlett JG, Campbell GD, Dean NC et al. Infectious Diseases Society of America/American Thoracic Society Consensus Guidelines on the management of community-acquired pneumonia in adults. Clin Infect Dis 2007; 44 (Suppl 2): S27-72.

16. British Thoracic Society Standards of Care Committee. British Thoracic Society Guidelines for the management of community-acquired pneumonia in adults. Thorax 2001; 56 (Suppl IV): 1-64.

17. Macfarlane J, Boswell T, Douglas G, Finch R, Holmes B, Honeybourne D et al. British Thoracic Society Guidelines for the management of communityacquired pneumonia in adults - 2004 update. www.brit-thoracic.org.uk/guidelines.

18. British Thoracic Society Pneumonia Guidelines Committee. The British Thoracic Society Guidelines for the management of community-acquired pneumonia in adults 2009 update. www.brit-thoracic.org.uk/guidelines.

19. Sociedad Chilena de Enfermedades Respiratorias. Consenso nacional en neumonías adquiridas en la comunidad en adultos y niños. Rev Chil Enf Respir 1999; 15: 67-136.

20. Sociedad Chilena de Enfermedades Respiratorias y SocieDAD Chilena de InFeCtología. Neumonía del adulto adquirida en la comunidad - Consenso Chileno 2005. Rev Chil Infect 2005; 22 (Supl 1): S7-S74.

21. Díaz A, Kuzmanic G, Platzer L, Sanfuentes F, Espinoza MA, SAldías F. Utilidad clínica del tratamiento antibiótico de la guía de la Sociedad Chilena de Enfermedades Respiratorias para la neumonía comunitaria en adultos hospitalizados. Rev Méd Chile 2003; 131: 847-56.

22. Pavié J, De la Prida JM, Díaz F, Saldías F. Manejo ambulatorio de la neumonía comunitaria del adulto en las unidades de emergencia. Servicio de Salud Viña del Mar-Quillota de la V Región. Rev Méd Chile 2005; 133: 1322-30.

23. Fang GD, Fine M, Orloff J, Arisumi D, Yu VL, Kapoor W ET AL. New emerging etiologies for communityacquired pneumonia with implications for therapy. A prospective multicenter study of 359 cases. Medicine (Baltimore) 1990; 69: 307-16.

24. Ruiz M, Ewig S, Marcos ma, Martínez Ja, Arancibia F, Mensa J, Torres A. Etiology of community-acquired pneumonia: impact of age, comorbidity, and severity. Am J Respir Crit Care Med 1999; 160: 397-405.

25. Neill AM, Martin IR, Weir R, Anderson R, Chereshsky A, Epton MJ ет Al. Community-acquired pneumonia: aetiology and usefulness of severity criteria on admission. Thorax 1996; 51: 1010-6.

26. Fine MJ, Auble Te, Yealy DM, Hanusa BH, Weissfeld LA, Singer DE et al. A prediction rule to identify low risk patients with community-acquired pneumonia. N Engl J Med 1997; 336: 243-50.

27. Saldías F, Mardónez JM, Marchesse M, Viviani P, Farías G, Díaz A. Neumonía adquirida en la comunidad en el adulto hospitalizado. Cuadro clínico y factores pronósticos. Rev Méd Chile 2002; 130: 1373-82.

28. Dintrans K, Andrade CL, Sánchez Je, Mendoza J. Neumonía adquirida en la comunidad en adultos, en el curso de la campaña de invierno 2003 en el Hospital San Juan de Dios. Rev Chil Enf Respir 2005; 21: 15-22.

29. Riquelme R, Riquelme M, Rioseco ML, Gómez V, Gil R, Torres A. Etiología y factores pronósticos de la neumonía adquirida en la comunidad en el adulto hospitalizado, Puerto Montt, Chile. Rev Méd Chile 2006; 134: 597-605.

30. Gil R, Undurraga A, Saldías F, Jiménez P, Barros M et AL. Estudio multicéntrico de factores pronósticos en adultos hospitalizados por neumonía adquirida en la comunidad. Rev Méd Chile 2006; 134: 1357-66.

31. Díaz A, Barría P, Niederman M, Restrepo Mi, Dreyse J, Fuentes G ET AL. Etiology of community-acquired pneumonia in hospitalized patients in Chile: the increasing prevalence of respiratory viruses among classic pathogens. Chest 2007; 131: 779-87.

32. Dean NC, Silver MP, Bateman KA, James B, Hadlock CJ, Hale D. Decreased mortality after implementation of a treatment guideline for community-acquired pneumonia. Am J Med 2001; 110: 451-7.

33. Menéndez R, Torres A, Zalacaín R, Aspa J, MartínVillasclaras JJ, Borderías L et al. Guidelines for the treatment of community-acquired pneumonia: predictors of adherence and outcome. Am J Respir Crit Care Med 2005; 172: 757-62.

34. Dean NC, Bateman KA, Donnelly SM, Silver MP, Snow GL, HALE D. Improved clinical outcomes with utilization of a community-acquired pneumonia guideline. Chest 2006; 130: 794-9.

35. Menéndez R, Reyes S, Martínez R, De la Cuadra P, VAllés M, Vallterra J. Economic evaluation of adherence to treatment guidelines in nonintensive care pneumonia. Eur Respir J 2007; 29: 751-6.

36. Dambrava PG, Torres A, Vallès X, Mensa J, Marcos Ma, Peñarroja G ET AL. Adherence to guidelines' empirical antibiotic recommendations and community-acquired pneumonia outcome. Eur Respir J 2008; 32: 892-901.

37. Ramírez Ja, Vargas S, Ritter GW, Brier ME, Wright A, SMITH S ET AL. Early switch from intravenous to oral antibiotics and early hospital discharge. A prospective observational study of 200 consecutive patients with community-acquired pneumonia. Arch Intern Med 1999; 159: 2449-54.

38. Pavié J, De la Prida JM, Díaz A, Saldías F. Manejo de la neumonía comunitaria del adulto en el Servicio de Salud Viña del Mar-Quillota. Abril-septiembre de 2003 Rev Chil Enf Respir 2006; 22: 13-20. 